\title{
An Unusual Case of Diffuse Alveolar Hemorrhage as a Clinical Manifestation of Atypical Hemolytic Uremic Syndrome: A Case Report
}

\author{
Ahmad Al-Shyoukh ${ }^{1}$, Moustafa Younis ${ }^{1}$, Omar Abughanimeh ${ }^{1}$, Mohammad Tahboub ${ }^{1}$, \\ Majdi S. Hamarshi ${ }^{2}$ \\ 1. Internal Medicine, University of Missouri-Kansas City 1 Saint Luke's Health System, Kansas City, USA \\ 2. Critical Care, University of Missouri-Kansas City 1 Saint Luke's Health System, Kansas City, USA
}

$\square$ Corresponding author: Ahmad Al-Shyoukh , alshyoukha@umkc.edu

Disclosures can be found in Additional Information at the end of the article

\section{Abstract}

Hemolytic uremic syndrome (HUS) is a constellation of microangiopathic hemolytic anemia, thrombocytopenia, and acute renal injury. HUS is subcategorized into primary or secondary HUS. Primary HUS is synonymous with atypical HUS (aHUS) and is attributed to genetic complement deficiency. Diffuse alveolar hemorrhage (DAH) is a serious condition complicating multiple systemic conditions. aHUS presenting as DAH is exceedingly rare. In this case, we present a 75-year-old male patient who presented with generalized weakness, malaise, and hemoptysis. He was found to have hemolytic anemia and thrombocytopenia, with elevated creatinine. Bronchoscopy confirmed DAH. He was started on plasmapheresis with a suboptimal response. aHUS was suspected and the patient was started on eculizumab with subsequent laboratory and clinical improvement. HUS and aHUS can present as DAH. It is very important to recognize both conditions as both are life threatening with high morbidity and mortality.

Received 06/21/2019

Review began 06/22/2019

Review ended 06/22/2019

Published 07/01/2019

CC Copyright 2019

Al-Shyoukh et al. This is an open access article distributed under the terms of the Creative Commons Attribution License CC-BY 3.0., which permits unrestricted use, distribution, and reproduction in any medium, provided the original author and source are credited.
Categories: Oncology, Pulmonology

Keywords: hemolytic uremic syndrome, atypical hemolytic uremic syndrome, diffuse alveolar hemorrhage, eculizumab, campylobacter jejuni

\section{Introduction}

Hemolytic uremic syndrome (HUS) is a condition that falls under the umbrella of thrombotic microangiopathies (TMA). It presents with microangiopathic hemolytic anemia, thrombocytopenia, and acute renal impairment [1-2]. HUS is traditionally classified into diarrhea positive or typical HUS that is preceded by certain infections such as Shiga-toxinproducing Escherichia coli (STEC) and atypical HUS (aHUS) that is attributed to genetic dysregulation of the alternative complement pathway [2-3]. Previously, aHUS was also used to refer to any HUS case that was not caused by STEC. More recently, HUS is classified as either primary HUS, synonymous with aHUS and complement-mediated HUS, which is due to genetic alterations of the alternative complement pathway or secondary HUS that is attributed to infections, malignancy, autoimmune conditions, hypertensive emergencies, pregnancy or drugs [4]. Though aHUS predominantly presents in the pediatric population, it has also been identified in adults [4-5]. Though unknown in the adult population, the estimated prevalence of complement-mediated aHUS in the pediatric population is seven per one million [6].

Diffuse alveolar hemorrhage (DAH) is a life-threatening syndrome that presents with 
hemoptysis, anemia, hypoxia and diffuse alveolar infiltrates [7-8]. A variety of diseases, including hematological, infectious, or immunological etiologies, are associated with the development of DAH. Idiopathic thrombocytopenic purpura and thrombotic thrombocytopenic purpura (TTP) are well-known causes of DAH resulting from vasculitis or capillaritis

[8]. However, HUS and specifically aHUS manifesting as DAH is exceedingly rare. It is important to distinguish aHUS from other TMA as management and prognosis vary [3]. The mainstay of treatment is supportive therapy along with eculizumab, a monoclonal recombinant antibody that inhibits complement factor five, which has been proven to treat and prevent recurrences of aHUS [9]. Herein, we illustrate the morbidity and management of a rare case of Campylobacter gastroenteritis triggering aHUS that presented with DAH.

\section{Case Presentation}

A 75-year-old male with a past medical history of rheumatoid arthritis, controlled on methotrexate and adalimumab, presented to an outside hospital with diarrhea, intermittent bright red blood with stool, and decreased oral intake. He was hypotensive (70/46 mm Hg) and tachycardic at 103 beats per minute (bpm). The rest of his vitals were otherwise unremarkable. His physical exam was only remarkable for mild right upper quadrant abdominal tenderness. His labs were significant for an elevated white blood cell (WBC) count of $22.5 \mathrm{x}$ $103 / \mathrm{mm}^{3}$ and an acute kidney injury (AKI) with a serum creatinine of $1.83 \mathrm{mg} / \mathrm{dl}$. His hemoglobin, platelet count, electrolytes, and liver function tests were all within normal limits. The patient was admitted to the floor for further management. He was started on intravenous (IV) fluids and completed a course of azithromycin with significant improvement in his symptoms. His polymerase chain reaction (PCR) stool studies were eventually positive for Campylobacter jejuni. On hospital day four, he was discharged. Upon discharge, his hemoglobin was $10.6 \mathrm{~g} / \mathrm{dl}$, platelets were $236 \times 10^{3} / \mathrm{mm}^{3}$, WBC count was $11.7 \times 10^{3} / \mathrm{mm}^{3}$ and creatinine was $0.90 \mathrm{mg} / \mathrm{dl}$.

Five days later, our patient presented to our institution with progressively worsening generalized weakness, fatigue, nausea, vomiting, intermittent shortness of breath and hemoptysis. The hemoptysis was small in amount, bright red in color without blood clots. He denied any chest pain, fever, or chills. His presenting vital signs were a heart rate of $96 \mathrm{bpm}$, blood pressure (BP) of 136/69 mm Hg, and oxygen saturation of $94 \%$ on room air. His physical exam was noteworthy for conjunctival pallor, scattered ecchymosis and petechia and bilateral lower extremity edema. Patient's labs were significant for a drop in his hemoglobin and platelet count to $7.1 \mathrm{~g} / \mathrm{dl}$ and $56 \times 10^{3} / \mathrm{mm}^{3}$, respectively. His reticulocyte count was elevated at $9.2 \%$ along with an elevated lactate dehydrogenase level (5454 U/L), a low haptoglobin level (< 10 $\mathrm{mg} / \mathrm{dl}$ ), and an elevated total and indirect bilirubin of $2.2 \mathrm{mg} / \mathrm{dl}$ depicting acute hemolytic anemia. The patient also demonstrated worsening renal function with a blood urea nitrogen of $66 \mathrm{mg} / \mathrm{dl}$ and serum creatinine of $3 \mathrm{mg} / \mathrm{dl}$. He had a normal international normalized ratio (INR) and elevated fibrinogen level (451 mg/dl). His chest X-ray showed bilateral pulmonary opacities mostly within lung bases. Given the concern for microangiopathic hemolytic anemia and multiorgan failure, he was admitted to the intensive care unit (ICU). Schistocytes were visualized on his peripheral smear. The hematology and oncology team was consulted and plasmapheresis was initiated given the possibility of TTP.

On hospital day (HD) two, the patient developed acute hypoxic respiratory failure with oxygen saturations as low as $86 \%$ on room air requiring oxygen supplementation with the nasal cannula at 3 liters/minute. Worsening bilateral perihilar heterogenous opacities were observed on the patient's CT scan of the chest without contrast (Figure 1). He was started empirically on vancomycin, piperacillin-tazobactam, and ciprofloxacin. Flexible bronchoscopy with sequential bronchoalveolar lavage showed that lavage aliquots are progressively more hemorrhagic, highly suggestive of DAH. On HD three, the patient was started on methylprednisolone 62.5 mg every 


\section{Cureus}

6 hours, due to concerns for vasculitis. Further workup revealed negative antineutrophil antibody (ANA), antineutrophil cytoplasmic antibody (ANCA), anti-glomerular basement membrane (Anti-GBM), and stool Shiga toxin one and two. Complement levels of C3, C4, and ADAMTS13 (a disintegrin and metalloprotease with thrombospondin type 1 repeats-13) activity level (90\%) were within normal limits.

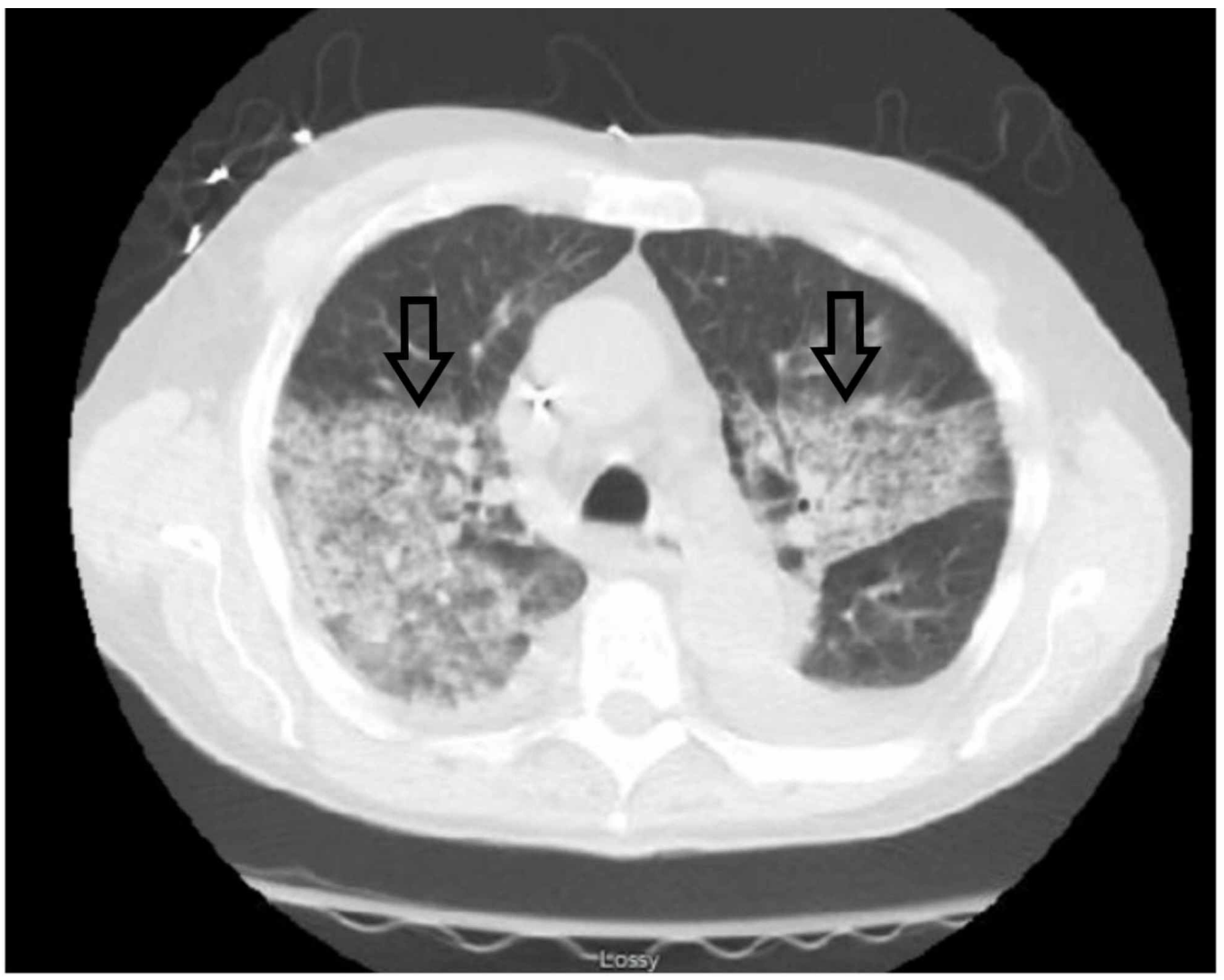

FIGURE 1: Computed tomography scan of the chest without contrast demonstrating bilateral perihilar heterogeneous opacities

The patient underwent a total of eight sessions of plasmapheresis and received multiple blood transfusions with minimal improvement in his hemoglobin and platelet count. Subsequently, atypical HUS labs screening for genetic mutations and antibodies to complement factors were sent and he was started on eculizumab $900 \mathrm{mg}$ intravenously once weekly. His methylprednisolone was switched to an oral prednisone taper. He was discharged on HD 16 after his second dose of eculizumab. The patient was discharged home to continue eculizumab $900 \mathrm{mg}$ IV once weekly as an outpatient. He received two more doses of eculizumab $900 \mathrm{mg}$ IV and it was switched to eculizumab $1200 \mathrm{mg}$ once every two weeks, which patient continues to receive to date. During his two months follow up, the patient's clinical condition and laboratory findings significantly improved (Table 1). Further, his final aHUS workup that was drawn after plasmapheresis was unremarkable with the exception of a low factor $\mathrm{H}$ complement antigen $21.6 \mathrm{mg} / \mathrm{dl}$ (normal range: 23.6-43.1) and a high SC5b-9 complement 330ng/ml (normal range $<251)$. 


\section{Cureus}

\begin{tabular}{|c|c|c|c|c|c|c|}
\hline $\begin{array}{l}\text { Significant } \\
\text { event }\end{array}$ & $\begin{array}{l}\text { Hospital } \\
\text { Day }\end{array}$ & $\begin{array}{l}\text { Hemoglobin } \\
\text { (g/dl) }\end{array}$ & $\begin{array}{l}\text { Hematocrit } \\
(\%)\end{array}$ & $\begin{array}{l}\text { Platelet count } \\
\times 10^{3} / \mathrm{mm} 3\end{array}$ & $\begin{array}{l}\text { Lactate dehydrogenase } \\
\text { level (U/L) }\end{array}$ & $\begin{array}{l}\text { Creatinine } \\
\text { (mg/dl) }\end{array}$ \\
\hline \multirow[t]{8}{*}{$\begin{array}{l}\text { Plasmapheresis } \\
\text { initiated }\end{array}$} & Day 1 & 6.4 & 19 & 67 & 5535 & 3.1 \\
\hline & Day 2 & 7.6 & 21 & 88 & 2351 & 2.8 \\
\hline & Day 3 & 8.1 & 23 & 147 & 1843 & 3.2 \\
\hline & Day 4 & 6.6 & 19 & 130 & 1091 & 3.1 \\
\hline & Day 5 & 7.5 & 22 & 98 & 1024 & 2.9 \\
\hline & Day 6 & 6.9 & 21 & 86 & 1105 & 2.6 \\
\hline & Day 7 & 7.4 & 22 & 83 & 1071 & 2.7 \\
\hline & Day 8 & 7.5 & 22 & 77 & 1067 & 2.5 \\
\hline \multirow{7}{*}{$\begin{array}{l}\text { Eculizumab } \\
\text { initiated }\end{array}$} & Day 9 & 7.3 & 22 & 80 & 1080 & 2.3 \\
\hline & Day 10 & 7.5 & 22 & 91 & 1614 & 2.5 \\
\hline & Day 11 & 7.1 & 21 & 92 & 1899 & 2.4 \\
\hline & Day 12 & 7.1 & 22 & 104 & 2051 & 2.2 \\
\hline & Day 13 & 7.5 & 23 & 104 & 2228 & 2.1 \\
\hline & Day 14 & 7.3 & 22 & 131 & 2120 & 2.1 \\
\hline & Day 15 & 6.7 & 20 & 122 & 1988 & 2.1 \\
\hline \multirow[t]{3}{*}{ Discharge } & Day 16 & 7.5 & 22 & 128 & 1955 & 2.2 \\
\hline & Day 60 & 12.4 & 37 & 274 & 670 & 1.8 \\
\hline & Day 150 & 14 & 41 & 241 & 453 & 1.4 \\
\hline
\end{tabular}

\section{TABLE 1: Laboratory values}

\section{Discussion}

We present a rare case of a 75-year-old male patient who came in with generalized weakness, fatigue, and hemoptysis preceded by an episode of Campylobacter diarrhea. It was associated with thrombotic microangiopathy and diffuse alveolar hemorrhage confirmed by bronchoscopy. He was managed with plasmapheresis initially without significant clinical improvement, later necessitating treatment with eculizumab. The presentation is significantly rare, and to the best of our knowledge, there are only two reported cases of Campylobacter-associated HUS associated with pulmonary hemorrhage documented in English literature [10-11].

aHUS refers to HUS cases due to genetic or acquired dysregulation of the alternative 
complement system pathway and coagulation cascade [1-2]. It is also referred to as primary or complement-mediated HUS. HUS results from endothelial cell injury, mainly in the renal microvasculature, resulting in the formation of fibrin and platelet thrombi. This injury can result from several mechanisms depending on the etiology of the inciting event. The formation of these thrombi leads to shearing of red blood cells and consequently the formation of schistocytes. Thrombocytopenia results from consumption of platelets in thrombi formation [12]. In aHUS, genetic or acquired dysregulation of the alternative pathway of the complement cascade in addition to an external trigger, such as Campylobacter in our case, leads to the formation of membrane attack complex C5b-9, which binds to endothelial cells' surface causing cell damage and swelling, thus creating a nidus for thrombi formation [2,12]. Either autoantibodies against Factor $\mathrm{H}$ or mutations in genes coding complement regulatory proteins, such as Factor H (CFH), Factor I (CFI), membrane cofactor protein (MCP), complement 3 (C3), FactorB (FB), or thrombomodulin contribute to this dysregulation [9]. This highlights the role of eculizumab in the management of aHUS: a monoclonal antibody that binds to complement protein $\mathrm{C} 5$ and then blocks the formation of C5 convertase, which ultimately leads to inhibition of the alternative complement pathway. aHUS cases are usually triggered by an infection. Keithlin et al. performed a metanalysis reviewing chronic sequelae of Campylobacter jejuni infection, with three out of the 31 studies reporting on the incidence of Campylobacter-induced HUS [13]. The first study encompassing a pediatric population did not have any reported cases of HUS, while the other two surveillance-based studies reported estimates of $0.005 \%$ and $0.003 \%$ [13].

aHUS can present at any age [5]. A study done in France by Fremeaux-Bacchi et al. evaluating 214 patients with aHUS showed that $58.4 \%$ of the affected individuals were adults [14]. Patients with HUS present with non-specific symptoms such as fatigue, malaise, shortness of breath, anorexia, decreased urine output and edema [1-2]. There have been some reported cases of aHUS presenting as DAH, but more data is needed to estimate the actual incidence [15]. In our case, and in other similar reported cases, it is unclear what is the exact mechanism of diffuse alveolar hemorrhage in patients with HUS; however, thrombocytopenia and thrombi formation in pulmonary vasculature could be contributing factors.

The diagnosis of aHUS is challenging as it resembles other TMA conditions including TTP. The diagnosis is usually suspected when patients present with the triad of microangiopathic hemolytic anemia, thrombocytopenia and acute renal injury [9]. In our patient, after recognizing a TMA, the first step was to exclude TTP. The distinction between HUS and TTP can be done based on ADAMTS13 activity [2]. However, ADAMTS13 levels are not usually available at the time of decision making thus empiric management, such as in our case, with plasmapheresis is vital. Also, the diagnosis of aHUS is mostly done after the exclusion of other TMA conditions [5]. We subsequently attempted to rule out Shiga toxin-mediated HUS and other potential secondary etiologies that might trigger HUS. Despite the first sessions of plasmapheresis, our patient's platelet counts remained unchanged. Given the exclusion of secondary etiologies and the poor response to plasmapheresis; aHUS was highly suspected. The diagnosis of aHUS further requires the demonstration of either genetic mutations and/or antibodies to complement factors [16]. These tests are not widely available and take weeks to result [9]. Despite the lack of family history, we elected to proceed with further aHUS work up given the poor clinical course in the absence of an underlying etiology. In our patient, the low Factor H can be consistent with aHUS, however, with low C4 and high SC5b-9, We believe this is not reflective of what our patient had as these labs were drawn after multiple sessions of plasmapheresis [4].

HUS management is supportive including fluid and electrolyte replacement, blood transfusion and dialysis [1-2]. In aHUS, plasmapheresis was traditionally the mainstay of treatment. However, it does not address complement dysregulation [9]. Lack of clinical and laboratory response after five to seven sessions of plasmapheresis should warrant the initiation of 
eculizumab if available [9]. Studies have shown that eculizumab improves prognosis and survival [9]. The clinical improvement seen in response to eculizumab further confirms the diagnosis of aHUS. Testing for anti-CFH antibodies prior to eculizumab infusion is the only urgent complement investigation required, as plasmapheresis would be the treatment of choice in such cases [9]. Eculizumab is administered as an intravenous infusion with weight-based induction and maintenance dosing [9]. Eculizumab therapy can be discontinued in patients who have had a favorable clinical and laboratory response to therapy [9]. There is a paucity of data defining the optimal timing for infusion cessation; therefore, the decision to withdraw eculizumab therapy should be made in conjunction with a clinician with expertise in managing aHUS. Finally, the prognosis in aHUS is not favorable with a mortality rate of $25 \%$ during the acute phase [3,9]. Around, $50 \%$ of the patients may progress to the end-stage renal disease within a year $[3,9]$.

\section{Conclusions}

In conclusion, aHUS triggered by Campylobacter gastroenteritis presenting as DAH is rare and life-threatening. There have been some reported cases of aHUS presenting as DAH, but more data is needed to estimate the actual incidence. A high degree of clinical suspicion is necessary to establish diagnosis after ruling out other etiologies of TMA such as TTP. In comparison with other TMAs, management of aHUS with eculizumab is unique highlighting the importance of proper diagnosis. It is encouraged to report such cases and highlight the management to help set up the stage to formulate a unified consensus for treatment.

\section{Additional Information}

\section{Disclosures}

Human subjects: Consent was obtained by all participants in this study. Conflicts of interest: In compliance with the ICMJE uniform disclosure form, all authors declare the following:

Payment/services info: All authors have declared that no financial support was received from any organization for the submitted work. Financial relationships: All authors have declared that they have no financial relationships at present or within the previous three years with any organizations that might have an interest in the submitted work. Other relationships: All authors have declared that there are no other relationships or activities that could appear to have influenced the submitted work.

\section{References}

1. Cody EM, Dixon BP: Hemolytic uremic syndrome. Pediatr Clin North Am. 2019, 66:235-246. 10.1016/j.pcl.2018.09.011

2. Fakhouri F, Zuber J, Frémeaux-Bacchi V, et al.: Haemolytic uraemic syndrome. Lancet. 2017, 390:681-696. 10.1016/S0140-6736(17)30062-4

3. Abu Ghanimeh M, Abughanimeh O, Qasrawi A, et al.: Atypical hemolytic uremic syndrome: when the environment and mutations affect organ systems. A case report with review of literature. Am J Hosp Med. 2017, 1:2. 10.24150/ajhm/2017.011

4. Loirat C, Frémeaux-Bacchi V: Atypical hemolytic uremic syndrome. Orphanet J Rare Dis. 2011, 8:60. 10.1186/1750-1172-6-60

5. Sridharan M, Go RS, Willrich MAV: Atypical hemolytic uremic syndrome: review of clinical presentation, diagnosis and management. J Immunol Methods. 2018, 461:15-22. 10.1016/j.jim.2018.07.006

6. Taylor CM, Machin S, Wigmore SJ, et al.: Clinical practice guidelines for the management of atypical haemolytic uraemic syndrome in the United Kingdom. Br J Haematol. 2010, 148:3747.10.1111/j.1365-2141.2009.07916.x. Epub 2009 Oct 11

7. Martínez-Martínez MU, Oostdam DAH, Abud-Mendoza C: Diffuse alveolar hemorrhage in autoimmune diseases. Curr Rheumatol Rep. 2017, 19:27. 10.1007/s11926-017-0651-y

8. Ioachimescu OC, Stoller JK: Diffuse alveolar hemorrhage: diagnosing it and finding the cause . 
Cleve Clin J Med. 2008, 75:264-265. 10.3949/ccjm.75.4.258

9. Raina R, Krishnappa V, Blaha T, et al.: Atypical hemolytic-uremic syndrome: an update on pathophysiology, diagnosis, and treatment. Ther Apher Dial. 2019, 23:4-21. 10.1111/17449987.12763

10. Bowen EE, Hangartner R, Macdougall I: Campylobacter-associated hemolytic uremic syndrome associated with pulmonary-renal syndrome. J Gen Intern Med. 2016, 31:353-356.

10.1007/s11606-015-3403-6

11. Delans RJ, Biuso JD, Saba SR, et al.: Hemolytic uremic syndrome after Campylobacter-induced diarrhea in an adult. Arch Intern Med. 1984, 144:1074-1076. 10.1001/archinte.1984.00350170242039

12. Tsai HM: Atypical hemolytic uremic syndrome: beyond hemolysis and uremia . Am J Med. 2019, 132:161-167. 10.1016/j.amjmed.2018.08.011

13. Keithlin J, Sargeant J, Thomas MK, et al.: Systematic review and meta-analysis of the proportion of Campylobacter cases that develop chronic sequelae. BMC Public Health. 2014, 22:14. 10.1186/1471-2458-14-1203

14. Fremeaux-Bacchi V, Fakhouri F, Garnier A, et al.: Genetics and outcome of atypical hemolytic uremic syndrome: a nationwide French series comparing children and adults. Clin J Am Soc Nephrol. 2013, 8:554-562. 10.2215/CJN.04760512

15. Vimal K. Derebail, Palak Parikh, J. Charles Jennette, et al.: A rare cause of the pulmonaryrenal syndrome: a case of atypical haemolytic-uraemic syndrome complicated by pulmonary haemorrhage. NDT Plus. 2008, 1:417-419. 10.1093/ndtplus/sfn145

16. Goodship TH, Cook HT, Fakhouri F, et al.: Atypical hemolytic uremic syndrome and C3 glomerulopathy: conclusions from a "Kidney Disease: Improving Global Outcomes" controversies conference. Kidney Int. 2017, 91:539. 10.1016/j.kint.2016.10.005 